\title{
RABDOMIÓLISIS EN LA PRIMERA SEMANA DE ENTRENAMIENTO. REPORTE DE DOS CASOS
}

\author{
YURIET ALEXANDRA TIBADUIZA MOGOLLON ${ }^{1^{*}}$ \\ ${ }^{1 *}$ Médico cirujano. Universidad Pedagógica y Tecnológica De Colombia. \\ Tunja, Colombia. \\ *Correspondencia: Yuriet Alexandra Tibaduiza Mogollón. \\ alexandratibaduiza@hotmail.com
}

Recibido: Febrero 2 de 2015 Aceptado: Junio 30 de 2015

\begin{abstract}
Resumen
La rabdomiólisis es un síndrome causado por lesión al músculo estriado con liberación de contenido celular muscular a la circulación, puede causar acidosis láctica, hiperfosfatemia, coagulación intravascular diseminada, hiperkalemia, síndrome compartimental, falla renal, e incluso puede comprometer la vida del paciente. La triada de síntomas incluye mialgias, debilidad y como manifestación cardinal orina de color rojoachocolatado, frente a lo cual se debe establecer diagnóstico diferencial ante la aparente eritrocituria e interrogarse sobre el uso de fármacos que enrojezcan la orina. El desconocimiento clínico y la demora diagnóstica, retardan el tratamiento específico incrementando así la morbimortalidad. El diagnóstico se puede confirmar ágilmente demostrando niveles elevados de: creatinina fosfoquinasa (CPK) sérica, análisis metabólicos, mioglobinuria, análisis de las enzimas intracelulares transaminasa glutámicooxalacético/aspartato aminotransferasa (GOT/ASAT), transaminasa glutámico pirúvico/alanino aminotransferasa (GPT/ALAT). El tratamiento está encauzado a acelerar la recuperación, identificar y corregir cualquier causa reversible de lesión muscular al igual que prevenir y tratar las complicaciones, la infusión oportuna y agresiva de cristaloides es el pilar del tratamiento, el uso de manitol, bicarbonato y antioxidantes aún es controvertido. En este reporte de casos se muestran las manifestaciones clínicas típicas de rabdomiólisis en dos hombres durante la primera semana de entrenamiento, en quienes se precipitó esta condición por deshidratación, actividad física intensa, inadecuada vestimenta y exposición a temperatura ambiental elevada (mayor a $25^{\circ} \mathrm{C}$ ). Estos pacientes fueron rápidamente diagnosticados, y recibieron tratamiento oportuno por lo que no presentaron complicaciones, actualmente están asintomáticos.
\end{abstract}

Palabras claves: Rabdomiólisis, mioglobinuria, deshidratación, mialgia, transaminasas. 


\title{
RHABDOMYOLYSIS AT THE FIRST WEEK OF TRAINING. TWO CASES REPORT
}

\begin{abstract}
Rhabdomyolysis is a syndrome caused by breakdown of skeletal muscle with release of muscle cell contents into the bloodstream, may cause lactic acidosis, hyperphosphatemia, disseminated intravascular coagulation, hyperkalemia, compartment syndrome, renal failure, and may even jeopardize the patient's life. The triad of symptoms including myalgia, weakness and urine - chocolate - red color as a cardinal manifestation, against which must be set before the differential diagnosis erythrocyturia apparent and question the use of drugs that turn red urine. Clinical ignorance and delay in diagnosis slow down specific treatment. The diagnosis can be confirmed swiftly demonstrating elevated levels of serum creatine phosphokinase (CPK), metabolic analysis, myoglobinuria, analysis of intracellular glutamic oxaloacetic transaminase/aspartate aminotransferase enzymes (ALT/AST), glutamic pyruvic transaminase/alanine aminotransferase (GPT/ALAT). Treatment is aimed at accelerating recovery, identifying any reversible cause of muscle injury, prevent and treat complications, timely and aggressive crystalloid infusion is the mainstay of treatment, the use of mannitol, bicarbonate and antioxidants even is controversial. This case report shows the typical clinical manifestations of rhabdomyolysis in two men in the first week of training, in whom this condition was precipitated by dehydration, intense physical activity, inadequate clothing and exposure to high ambient temperature (above $25^{\circ} \mathrm{C}$ ). These patients were quickly diagnosed and received timely treatment so no present any complications, actually they are asymptomatic.
\end{abstract}

Keywords: Rhabdomyolisis, myoglobinuria, dehydration, mialgia, transaminases.

\section{RABDOMIÓLISE NA PRIMEIRA SEMANA DE TREINAMENTO. RELATO DE DOIS CASOS}

\begin{abstract}
Resumo
A rabdomiólise é uma síndrome causada por lesão do músculo estriado com libertação do conteúdo das células musculares para a torrente sanguínea, a suas causas são acidose láctica, hiperfosfatemia, coagulação intravascular disseminada, hipercalemia, síndrome do compartimento, insuficiência renal, e pode mesmo comprometer a vida do paciente. A tríade de sintomas inclui mialgias, fraqueza e, como manifestação cardinal, urina de cor vermelho - achocolatado, por isso é preciso estabelecer um diagnostico diferencial ante a eritrocitária aparente e questionar sobre os medicamentos que tornam a urina vermelha. A ignorância clínica e atraso de diagnóstico retardam o tratamento específico aumentando assim a morbidade $e$ mortalidade. O diagnóstico pode ser confirmado rapidamente demonstrando níveis elevados de: creatinina fosfoquinase (CPK), análises metabólicos, mioglobinúria, análises das enzimas intracelulares transaminase glutâmico-oxalacética/aspartato aminotransferase (TGO/AST), transaminase glutâmico pirúvica/alanina aminotransferase (TGP/ALT). O tratamento tem sido orientado para acelerar a recuperação, identificar e corrigir qualquer causa reversível de lesão muscular assim como prevenir e tratar complicações. A infusão oportuna e agressiva de cristaloides é a base do tratamento, a utilização de manitol, bicarbonato $e$ antioxidantes é ainda controversa. Neste informe de casos se revelam as manifestações clinicas tipicas de rabdomiólise em dois homens durante a primeira semana treinamento, em eles foi manifestada esta condição por desidratação, atividade física intensa, roupas inadequadas e exposição ao ambiente de alta temperatura (superior a 25). Estes pacientes foram rapidamente diagnosticados e eles receberam um tratamento oportuno pelo qual eles não presentaram complicações e atualmente eles não têm nenhum sintoma.
\end{abstract}

Palavras-chave: Rabdomiólise, mioglobinúria, desidratação, mialgia, transaminases. 


\section{Introducción}

La rabdomiólisis es un síndrome bioquímico y clínico debido al daño y necrosis en el músculo esquelético con la subsecuente liberación de contenido celular al torrente circulatorio de enzimas Creatinfosfoquinasa (CPK), lactato deshidrogenasa (LDH), aldolasa, transaminasa glutámico oxalacético (TGO), mioglobina (1), pigmentos, hemoglobina y electrolitos, potasio, fosforo y ácido úrico (2). El desconocimiento clínico y la demora diagnóstica, retardan el tratamiento específico incrementando así la morbimortalidad. La rabdomiólisis debe buscarse intencionalmente en los pacientes con historia de esfuerzo físico intenso, asociado a la triada clásica de síntomas: mialgias, debilidad y orina rojoachocolatado (dada por la presencia de Mioglobina aún en ausencia de hematuria).

El diagnóstico se puede confirmar ágilmente demostrando niveles elevados de creatinina fosfoquinasa (CPK) sérica, análisis metabólicos con hiperuricemia significativa, mioglobinuria, glucosuria transitoria, y elevación de las enzimas intracelulares: transaminasa glutámicooxalacètica/aspartato aminotransferasa (GOT/ASAT), transaminasa glutámico pirúvica/ alanino aminotransferasa (GPT/ALAT). El tratamiento está encauzado a acelerar la recuperación, identificar y corregir cualquier causa reversible de injuria muscular al igual que prevenir y tratar las complicaciones; la infusión oportuna y agresiva de cristaloides es el pilar del tratamiento, durante el tratamiento inicial generalmente se requieren 2 a $3 \mathrm{~L}$ de cloruro de sodio $0.9 \%$ (3) por hora y al alcanzar la estabilidad hemodinámica entre 300 a $500 \mathrm{~mL}$ por hora, algunos autores argumentan que esta infusión de cristaloides por si solos pueden producir suficiente diuresis de solutos y previene la progresión a falla renal (4), el uso de manitol, bicarbonato y antioxidantes aún es controvertido pues algunas publicaciones mencionan que no muestran beneficios adicionales (5). Un temprano diagnóstico y un adecuado tratamiento mejoran ampliamente el pronóstico y previenen el desarrollo de complicaciones renales crónicas.

A continuación se reporta rabdomiólisis en dos hombres durante la primera semana de entrenamiento, quienes presentaron las manifestaciones clínicas típicas de la enfermedad, debilidad, mialgias y orina rojo-achocolatado, tras la realización de entrenamiento físico intenso asociado a deshidratación por aumento en las perdidas insensibles de líquidos corporales así como déficit en la ingesta hídrica, vestimenta inadecuada para la realización de la actividad física (chaqueta y pantalón compuestos de algodón $60 \%$, poliéster $40 \%$, además de una camiseta en algodón, cubriendo todo el cuerpo excepto la cara, impidiéndose la liberación térmica), aunado a temperatura ambiental de $30^{\circ} \mathrm{C}$ aproximadamente. Éstos pacientes fueron rápida- mente diagnosticados, y recibieron tratamiento oportuno por lo que no presentaron complicaciones ni secuelas.

\section{Reporte de casos}

\section{Caso 1}

Paciente masculino de 21 años, sin antecedentes personales o familiares de importancia. Siete días antes de su ingreso al centro de atención el paciente inició actividad física intensa durante aproximadamente dos horas por día; además de flexiones de brazo realizó sentadillas, en aproximadamente diez series de 100 repeticiones, al terminar el ejercicio presentó dolor en las extremidades ejercitadas. Al interrogatorio dirigido refirió emisión de orina "negra", debilidad muscular en las piernas, mialgias y perdida de la fuerza muscular con dificultad para continuar el ejercicio físico iniciado desde la mañana.

En el examen físico se encontró paciente en buena condición general, consciente, orientado, álgico. Los signos vitales evidenciaron presión arterial de $116 / 76 \mathrm{mmHg}$, frecuencia cardíaca de 70 latidos por minuto, frecuencia respiratoria de 19 por minuto, temperatura de $37,6^{\circ} \mathrm{C}$, IMC: 23.89. Pupilas simétricas con adecuada respuesta a la luz. Sin ingurgitación yugular. Los campos pulmonares sin agregados, en la región precordial: ruidos rítmicos sin soplos. El abdomen blando, depresible, no doloroso a la palpación. Como hallazgo anormal se encontró hipersensibilidad en masa muscular de extremidades inferiores, cara anterior de ambos muslos y con fuerza muscular disminuida $4 / 5$, reflejos de estiramiento normales. Los análisis de laboratorio al ingreso fueron: citometría hemática normal, química sanguínea normal, incluyendo azoados. Concentraciones séricas de $\mathrm{K}, \mathrm{Cl}$ y $\mathrm{Na}$ normales. Las enzimas musculares ALT: 4220 U/L, AST: 1460 U/L, creatincinasa total $98320 \mathrm{UI} / \mathrm{L}$ (VN: 10-120 UI/L). El parcial de orina reportó densidad mayor de 1030, eritrocituria 30 x campo, cilindros granulosos $3 \mathrm{x}$ campo. Se consideró el diagnóstico de rabdomiólisis de esfuerzo (secundaria a deshidratación y esfuerzo físico), se inició tratamiento con solución salina al $0.9 \%$ y diuresis alcalina con bicarbonato de sodio. El paciente evolucionó adecuadamente con descenso de la concentración de enzimas musculares. Se egresó cinco días después con indicación de beber líquidos en abundancia y consultar nuevamente si reiteradamente presentaba orina turbia. Control paraclínico en 1 mes. En la Tabla 1 se ven resultados de hospitalización.

\section{Caso 2.}

Paciente masculino de 18 años. Antecedentes personales tóxicos: consumo esporádico de marihuana; sin anteceden- 
tes familiares de importancia. Cuatro días antes de su ingreso al centro de atención el paciente inició actividad física durante aproximadamente tres horas por día, practicó sentadillas con aproximadamente 20 libras de peso en aproximadamente diez series de 100 repeticiones y trote de resistencia, al terminar el ejercicio presentó dolor en miembros inferiores. Al interrogatorio dirigido refirió emisión de orina "negra", mialgias en los gemelos y muslos.

En el examen físico se encontró al paciente en buena condición general, consciente, orientado. Los signos vitales evidenciaron presión arterial de $118 / 72 \mathrm{mmHg}$, frecuencia cardíaca de 78 latidos por minuto, frecuencia respiratoria de 20 por minuto y temperatura de $38,2^{\circ} \mathrm{C}$, IMC: 23.46 . Pupilas simétricas con adecuada respuesta a la luz. Sin ingurgitación yugular. Los campos pulmonares sin agregados, la región precordial con ruidos arrítmicos sin soplos. El abdomen blando, depresible, no doloroso a la palpación. Como hallazgo anormal se encontró hipersensibilidad en masa muscular de gastrocnemios y muslos, fuerza muscular bilateral 5/5, reflejos normales. Los análisis de laboratorio al ingreso fueron: citometría hemática normal, química sanguínea normal, incluyendo azoados, concentraciones séricas de $\mathrm{K}, \mathrm{Cl}$ y $\mathrm{Na}$ normales. Las enzimas musculares ALT: $910 \mathrm{U} / \mathrm{L}$, AST: $2480 \mathrm{U} / \mathrm{L}$, creatincinasa total $122784 \mathrm{UI} / \mathrm{L}$ (VN: 10-120 UI/L). El examen general de orina reportó densidad mayor de 1030, eritrocituria 1012 x campo, cilindros granulosos 2 x campo. Se estableció el diagnóstico de rabdomiólisis de esfuerzo (secundaria a deshidratación y esfuerzo físico).

El paciente recibió tratamiento con infusión endovenosa de solución salina al 0.9\%, 2000 cc en bolo, seguido de 200 cc/ hora, hasta evidenciar orina clara, la evolución indicó mejoría con disminución de enzimas musculares. Egreso satisfactorio cuatro días después, con indicación de solicitar valoración nueva en caso de presentar orina marrón-rojiza, ingesta abundante de líquidos, se ordenó control paraclínico en 1 mes. En la tabla 1 aparecen resultados de paraclínicos.

Tabla 1. Evolución de los resultados de laboratorio. En paciente 1 y 2

\begin{tabular}{|c|c|c|c|c|c|c|c|c|c|}
\hline & & Pacier & & & & aciente & & & PARAMETROS \\
\hline & Ingreso & $\begin{array}{l}\text { Día } \\
2\end{array}$ & Día 3 & Día 4 & Ingreso & Día 1 & Día 2 & Día 3 & \\
\hline $\operatorname{ALT}(\mathrm{U} / \mathrm{L})$ & 4220 & & 460 & 358 & 910 & & & 410 & $10-35 \mathrm{U} / \mathrm{L}$ \\
\hline AST(U/L) & 1460 & & 1011 & & 2480 & & & 375 & $<35 \mathrm{UI} / \mathrm{L}$ \\
\hline CPK TOTAL & 98320 & & 48534 & 15152 & 122784 & & & 6435 & $55-170 \mathrm{U} / \mathrm{L}$ \\
\hline $\mathrm{Na}$ & 142 & & & 141.7 & 138 & & & 142.2 & $134-144 \mathrm{mmol} / \mathrm{L}$ \\
\hline $\mathrm{Cl}$ & 104 & & & 104.7 & 112 & 109 & & 105 & $98-107 \mathrm{mEq} / \mathrm{L}$ \\
\hline $\mathrm{K}$ & 4.6 & & & 4.03 & 5 & 4.8 & & 3.9 & 3.6-5.0mEq/L \\
\hline $\mathrm{Hb}(\mathrm{g} / \mathrm{dl})$ & 13.8 & 14.2 & 14.5 & 13.0 & 13.9 & & 13.9 & & $13.2-17.5 \mathrm{~g} / \mathrm{dl}$ \\
\hline $\begin{array}{l}\text { Leucocitos } \\
\text { (miles) }\end{array}$ & 7,3 & 5,2 & 4,8 & 5,6 & 8,1 & & 7,2 & & \\
\hline Densidad & 1030 & 1020 & & 1010 & 10320 & & 1000 & & $1010-1030$ \\
\hline $\mathrm{pH}$ & 6 & & & & 6 & 5.5 & & & $4.5-8$ \\
\hline Eritrocitos & $30 \mathrm{p} / \mathrm{c}$ & & Negat & Negat & $\begin{array}{l}10-12 \\
p / c\end{array}$ & $\begin{array}{l}5 \\
\mathrm{p} / \mathrm{c}\end{array}$ & Negat & Negat & 0 \\
\hline Glucosa & 90 & & & 80 & 88 & & Negat & Negat & 0 \\
\hline BUN & 7 & & & 10 & 16 & 14 & 13 & 9 & $5-20 \mathrm{mg} / \mathrm{dL}$ \\
\hline Urea & 32 & 24 & 20 & 19 & 33 & 28 & 25 & 19.5 & $20-40 \mathrm{mg} / 100 \mathrm{ml}$ \\
\hline Creatinina & 0.91 & 0.8 & & 0.81 & 1.3 & 1.1 & 1 & 1 & $0.6-1.2 \mathrm{mg} / \mathrm{dL}$ \\
\hline
\end{tabular}




\section{Rabdomiólisis}

La rabdomiólisis es una entidad con múltiples factores etiológicos, puede ser la primera manifestación de una enfermedad hereditaria del metabolismo muscular por alteraciones glucógeno (6), o puede deberse a entidades genéticas como distrofias musculares, miotonìa condro- displàsica, alteraciones metabólicas (7), inhalación de agentes anestésicos y relajantes musculares como: halotano, enflurano, isoflurano, suxamethoniom, suxethorium o decamethonium, (causantes de hipertermia maligna), consumo de cocaína y corea central (8) entre otros (Ver tabla 2) (9).

Tabla 2. Causas de Rabdomiolisis. Modificado de cita 9: Poels PJE, Gabreëls FJM. Rhabdomyolysis: a review of literature. Clin Neurol Neurosurg. 1993; 95:172-92.

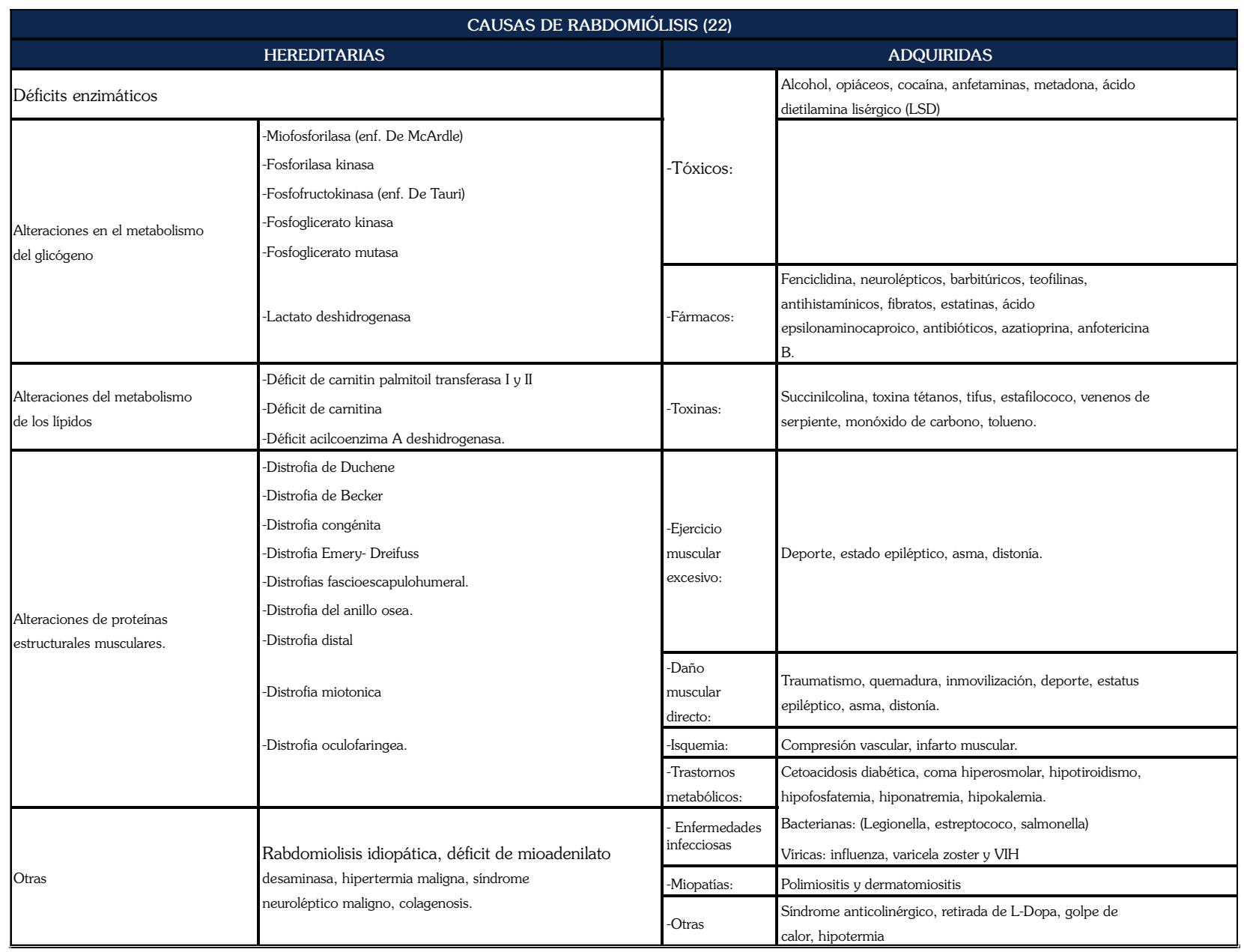

De ahí que al iniciar cualquier tipo de entrenamiento muscular es trascendental la prudencia para conseguir los objetivos atléticos planteados ya que ésta entidad se ha reportado tanto en sujetos sanos tras practicar ejercicios de baja intensidad (10), como en sujetos que recién inician el entrenamiento sometidos a exceso de actividad física, y en sujetos con entrenamiento rutinario tras actividad intensa sin coexistencia de otros factores (11); por citar un ejemplo se ha comunicado mioglobinemia en reclutas en la primera semana de entrenamiento (12).
En nuestros pacientes el factor precipitante fué la inadecuada ingesta hídrica antes de la realización de ejercicio (Paciente 1: un jugo de $150 \mathrm{cc}$ a la hora del desayuno y un jugo de 320 cc en la merienda; Paciente 2: aprox 300 cc de un caldo al desayuno, un tinto $130 \mathrm{cc}$, y una bolsa de jugo $320 \mathrm{cc}$ en la merienda) y el entrenamiento prolongado y extenuante.

El diagnóstico y tratamiento de la rabdomiólisis aún son retos importantes ya que cursan con un conjunto de 
síntomas y de signos similares a los de otras condiciones más comunes, dilatándose así el oportuno diagnóstico y tratamiento.

El inadecuado manejo de esta condición puede causar falla renal crónica o incluso la muerte.

\section{Epidemiología}

Se considera que entre el 10\% al 50\% de los pacientes con rabdomiólisis desarrollan falla renal aguda (13). La mortalidad en los pacientes que desarrollan falla renal aguda por rabdomiólisis se estima entre 7\% al 80\% (14) siendo aún mayor en los pacientes con disfunción orgánica múltiple (15).

\section{Lesión muscular.}

La lesión del músculo estriado causa aumento del flujo de agua, sodio y cloro al retículo sarcoplásmico (16), debido al estrés al que se ha sometido el miocito, causando inflamación por secuestro de líquidos en tercer espacio y autodestrucción. En el intercambio iónico con la salida del sodio intracelular se presenta entrada de iones de calcio libre en grandes cantidades, generando contracciones persistentes, agotando la energía y causando muerte celular. El calcio activa la fosfolipasa A2 incrementando la producción de radicales libres (17). Grupos de neutrófilos activados migran al sitio de la lesión, aumentando el daño por liberación de proteasas y extendiendo la necrosis muscular.

Carcelén y col destacaron la hinchazón, edema y dolor en los grupos musculares afectados, los cuales generalmente son el pectoral mayor, tríceps (18), pantorrilla y espalda baja (19).

\section{Fisiopatología}

El músculo necrótico libera grandes cantidades de mioglobina a la circulación, la mioglobina es una proteína hem pequeña de 151 aminoácidos del músculo estriado; en condiciones habituales en sujetos sanos, la mioglobina contenida en el musculo esquelético es de aproximadamente $4 \mathrm{mg} / \mathrm{g}$ de peso seco, su peso molecular es de aproximadamente 17000 por lo cual filtra fácilmente a través de los glomérulos y pasa a la orina, su concentración plasmática oscila de 0 a $0.003 \mathrm{mg} / \mathrm{dL}$ aproximadamente y el 50\% se encuentra ligado a la alfa2 globulina, la mioglobina contiene hierro en forma de óxido ferroso $(\mathrm{Fe} 2+)$ indispensable para la unión del oxígeno molecular. Se ha comprobado que la mioglobina ostenta un comportamiento similar a la enzima peroxidasa que induce oxidación desmandada de biomoléculas, peroxidación lipídica y generación de isoprostanos (20).
Greenhill y Gruskin en 1976 mencionaron que en grandes cantidades la mioglobina se asocia con insuficiencia renal aguda (21), la isquemia renal mediada por mioglobina incrementa el estrés oxidativo mediante el aumento de malondialdehido hepático (marcador de peroxidación lipídica) y disminuye los antioxidantes (superóxido dismutasa y glutatión total) promoviéndose y expandiéndose la inflamación, apoptosis y daño tisular sobre todo a nivel hepático y expandiendo la disfunción multisistémica (22). Los mecanismos conocidos hasta el momento son la activación leucocitaria con la respectiva infiltración a órgano afectado, cambios en los niveles de factores solubles como citocinas, quimiocinas y regulación de la muerte celular en los órganos extrarenales(23), en estudios animales se ha demostrado que la falla renal aguda repercute de manera fehaciente en órganos distantes(24).

El umbral renal para la mioglobina es de 0.5 a $1.5 \mathrm{mg} / \mathrm{dL}$, motivo por el que aparece en la orina cuando la concentración plasmática excede el límite superior, la concentración urinaria debe exceder los $100 \mathrm{mg} / \mathrm{dL}$ para pigmentar la orina y dar el particular aspecto rojo café lodoso.

\section{Fundamentos de fisiología térmica, alteraciones térmicas durante el ejercicio.}

En condiciones habituales los procesos fisiológicos trabajan de manera sincronizada manteniendo la presión venosa, la función muscular, la regulación hídrica, y la regulación térmica (25) mediante la vasoconstricción en el tejido inactivo y la vasodilatación en el músculo y la piel, pero en ambientes cálidos estos mecanismos disminuyen la capacidad de respuesta por lo que puede presentarse hipertermia, deterioro físico, y golpe de calor, favorecidos por la deshidratación (26) causada por el aumento de las de pérdidas por perspiración, aumento de la transpiración y la falta de ingesta liquida.

Al detectarse aumentos bruscos de temperatura corporal los mecanismos disipadores de los que se vale el individuo para mantener su balance térmico se activan: conducción, convección, evaporación y radiación (27).

\section{De modo que:}

\section{Temperatura total $=$ temperatura producida - temperatura disipada.}

$$
\mathrm{G}=(\mathrm{Pt}-\mathrm{W}) \pm \mathrm{C} \pm \mathrm{K} \pm \mathrm{R}-\mathrm{E}
$$

Donde $\mathrm{G}$ es el gasto cardiaco, Pt es la producción térmica, $\mathrm{W}$ es el trabajo realizado, y C, K, R y E representan: convección, conducción, radiación y evaporación respectivamente. Vale la pena destacar que los tres primeros son 
mecanismos tanto de pérdida como de ganancia, mientras que el último exclusivamente produce pérdida de calor.

En ambientes calurosos la evaporación será el mecanismo valioso y principal de perdida de calor evitando el calentamiento del núcleo interno por encima de los $30^{\circ} \mathrm{C}$, ocasionando graves efectos si la capacidad de producción del sudor se limita o disminuye la cantidad de agua disponible para la evaporación en piel.

La distribución de las 2.500.000 glándulas sudoríparas sometidas al control colinérgico en la superficie corporal facilitan la evaporación; el sudor al pasar de fase liquida a gaseosa, absorbe la energía térmica de la superficie cutánea y produce enfriamiento, así mismo la evaporación obedece a la humedad del ambiente. En un ambiente húmedo el sudor se extiende por un área mayor de piel antes de evaporarse, mientras en un ambiente seco la mayor parte del sudor se volatiliza, motivo por el cual un individuo siente más calor en un ambiente húmedo que en uno seco.

Sawka notó la intolerancia al calor ambiental en más de la mitad de los sujetos deshidratados cuando las pérdidas alcanzaron cerca del 8\% del peso corporal, (se considera deshidratación no grave si hay pérdida inferior al 4\% (28) del peso corporal). Estas cifras de deshidratación raramente son encontradas en deportistas excepto en los triatlonistas, corredores de largas distancias, y bicicrosistas, quienes por situaciones intrínsecas a sus disciplinas tienen dificultades para la hidratación, y en los que dada la importancia de la medición en tiempo real de las oscilaciones térmicas se promueve el uso de termómetros rectales.

\section{Líquidos corporales y variaciones durante el ejercicio.}

El cuerpo humano se compone aproximadamente de 65\% de agua, dividido en líquido extracelular (plasma y espacio intersticial) y liquido intracelular. El ejercicio y el aumento de temperatura con la deshidratación influyen en la redistribución hídrica corporal a causa de cambios en la presión hidrostática (29) y osmótica.

Teniendo en cuenta que el sudor es hipotónico con respecto al plasma, los deportistas cursan con hiperosmolaridad hipovolémica, ya que la deshidratación dada por altas temperaturas ambientales origina perdidas de líquido extracelular principalmente. La deshidratación ocasiona hipernatremia y a medida que evoluciona se compromete el espacio intracelular; se sospecha tercer espacio por lesión muscular cuando existen grandes pérdidas intravasculares en ausencia de otras circunstancias que puedan explicarla.

Considerándose que el cuerpo humano durante el ejercicio físico intenso puede perder hasta 15 litros diarios de sudor, durante el entrenamiento debe considerarse:

1) La pertinencia de la realización del ejercicio en ambientes calurosos.

2) El consumo adecuado de líquidos para evitar la deshidratación extracelular y el descenso del volumen circulante eficaz como fuente de anomalías microcirculatorias y facilitadoras de la isquemia muscular (30).

La deshidratación puede causar deterioro en la función renal por decremento de la perfusión, así como alteraciones en el sistema endocrino, en la regulación metabólica e incluso alteraciones de las concentraciones iónicas plasmáticas como respuesta al ejercicio (31), en el sistema gastrointestinal el decremento del lactato sérico origina disminución del flujo hepático a causa de la vasoconstricción en el lecho esplàcnico, con preservación del flujo al estómago y otros órganos intraabdominales incluyendo a los riñones; todo lo anterior para acrecentar el flujo sanguíneo a los grupos musculares sometidos a mayor trabajo, alterándose la distribución de oxígeno y facilitándose los trastornos en la remoción de desechos, dada la capacidad tope muscular de fatiga.

En cuanto al sistema cardiovascular, el gasto cardiaco aumenta para compensar la disminución del volumen por perdidas hídricas manteniendo la volemia, todo mientras se presenta vasodilatación cutánea para regular las temperaturas exógenas y endógenas (32) alcanzadas. En sujetos hipohidratados se altera la capacidad de mantenimiento de la presión de llenado, sacrificando la circulación periférica y redistribuyéndola a la circulación central. El incremento en la viscosidad sanguínea y la disminución del volumen que regresa al corazón a causa de las perdidas hídricas disminuye la presión de llenado y el volumen sistólico.

\section{Líquidos consumidos y deshidratación en el deportista.}

El agua no es el mejor líquido post ejercicio para reponer las perdidas por sudor dado su volumen y osmolaridad. El consumo de agua disminuye la osmolaridad plasmática, limitando la percepción de la sed e incrementando la producción de orina.

Algunas bebidas deportivas incluyen cafeína y otras xantinas, justificándose su adición por la estimulación al sistema nervioso central, incrementando el tiempo de percepción del cansancio e identificación de la sed, así como la mejoría aparente del desempeño durante el ejercicio aeróbico de larga duración (33), sin embargo estos compuestos poseen acción diurética (34), por lo que se discutiría su uso habitual, particularmente al encontrarse 
que el efecto de estas xantinas en deportes de intensidad elevada y de corta duración todavía es motivo de polémica (35). El efecto diurético de la cafeína ha sido observado con elevadas ingestas mayores a $642 \mathrm{mg}$ pero no con cantidades inferiores a $300 \mathrm{mg}$, sobre todo durante el reposo, además de aumentar la periodicidad miccional, exacerbando la deshidratación y la hipovolemia, secundario al bloqueo de la reabsorción renal de sodio, el aumento de la excreción de sodio y agua y la disminución de la excreción de K (36).

Otras publicaciones han manifestado que ni el uso de café ni de cafeína causan deshidratación o alteraciones en la regulación de los fluidos corporales (37) ni alteraciones hidroelectrolíticas (38), o hipertermia (39) durante el ejercicio, pues mencionan que las catecolaminas que se liberan al ejercitarse producen vasoconstricción de las arteriolas renales, reduciendo así la tasa de filtración glomerular y contrarrestando el efecto de la cafeína (40), de ahí que para recomendar o rechazar su uso aún faltan estudios.

Incluir sodio en los líquidos de rehidratación mejora la conservación del volumen (manteniendo los niveles de aldosterona y vasopresina bajos (41) y permitiendo así mantener mejor la percepción de sed, y la concentración urinaria. Una forma rápida de rehidratación consiste en agua fría $1.5 \mathrm{~L}$ con $2 \mathrm{~g} \mathrm{NaCl}$ por cada hora de ejercicio intenso (42) ó según la percepción de sed.

Incluir carbohidratos (43) a las soluciones rehidratantes ayuda a efectivizar la absorción intestinal de sodio y agua, además de suplir la necesidad de glucógeno (44). Se ha descrito que las bebidas frías, que son mezcla de carbohidratos y electrolitos son las predilectas y recuperan en mayor cantidad el peso perdido tras la actividad física, con respecto al agua pura a temperatura ambiente o tibia (45), la temperatura ideal de los líquidos debe oscilar entre 15 a $21^{\circ} \mathrm{C}$; las bebidas demasiado frías enlentecen la absorción y pueden causar desvanecimientos y lipotimias.

Experimentalmente se realizó una medición en 40 individuos (pesos corporales entre 64-70kg), ponderando el peso corporal antes y después de un entrenamiento físico de 60 minutos de intensidad, tras la realización de combinaciones de "trote" y flexiones de brazo en el suelo, squat (sentadillas), "polienchilenas", crunch (encogimientos abdominales) y elevaciones del tronco en el suelo, a una temperatura ambiental de $34^{\circ} \mathrm{C}$, en atuendo deportivo (camiseta en algodón y pantaloneta en poliéster) y encontramos que de los 40 individuos 31 presentaron perdida post ejercicio entre 1 a $2 \mathrm{~kg}$ de peso, con lo que se pudo corroborar que el estimado de la cantidad de sudor durante la actividad deportiva intensa alcanza valores iguales o superiores a 3litros/hora (46).

\section{Trabajo musculardesencadenante}

El cuerpo cuenta con mecanismos de contracción muscular especializados para dar respuesta a todas las necesidades y realizar variedad de movimientos. Ver tabla 3 (47). Knuttgen \& Kramer (48) definieron la fuerza como "el poder de contracción de los músculos como resultado de un solo esfuerzo máximo en un movimiento dado, a una velocidad específica", teniendo consecuencias particulares según el esfuerzo realizado. Los episodios se deben a los efectos aditivos de varios factores desencadenantes incluyendo sobre entrenamiento o factores idiopáticos (48).

En publicaciones anteriores de rabdomiólisis por esfuerzo físico (49), los autores manifiestan que para la célula muscular resulta más nocivo el trabajo excéntrico que el concéntrico ya que el esfuerzo realizado durante el estiramiento (alargamiento de la fibra muscular) causa más daño a la unidad muscular que el acortamiento en el trabajo concéntrico, todo esto por los mecanismos que comprometen la bomba Na-K ATPasa (50), y la lisis subsecuente de la fibra muscular con la correspondiente liberación y elevación sérica, superando hasta cinco veces el límite superior de lo normal (>1.000 U/1) (51) de los valores de la CPK.

En los casos presentados los tipos de contracciones musculares desencadenantes fueron combinaciones de contracciones auxotónicas excéntricas (sentadillas) y contracciones isométricas (flexión de brazos), de modo que, la rabdomiolisis puede presentarse posterior a la ejecución de actividad física en individuos "sanos", cuando la entrega energética muscular resulta ineficiente ò en sujetos desacondicionados que empiezan de forma no progresiva el entrenamiento muscular, sobretodo en climas húmedos y calurosos y también cuando el individuo está bajo efectos anticolinérgicos o usa ropa muy ajustada, que dificulta la sudoración; la hipokalemia resulta ser otro factor destacable (52). La elevación de las enzimas musculares y la mioglobinuria subclínicas son comunes después de un ejercicio físico intenso, elevando la $\mathrm{CK}$ hasta 16 veces en sujetos que corren ultra maratón (53).

Tabla 3. Esquema modificado de cita 50. Iriarte C. La fuerza. Entrenamiento para la salud y la estética. Grupo Sobre Entrenamiento; 42.

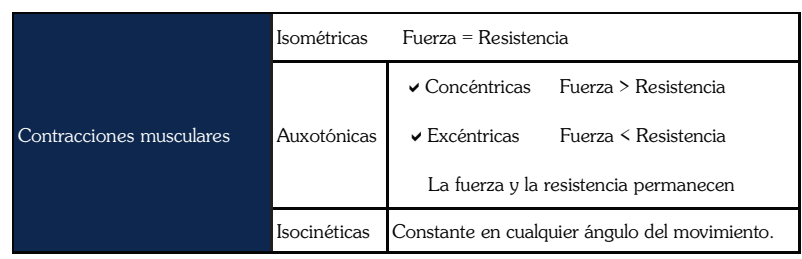




\section{Diagnóstico}

Si bien la evaluación bioquímica es la que permite el diagnóstico definitivo de la rabdomiolisis, los aspectos clave que facilitan su diagnóstico temprano son la aparición de síntomas musculares luego de un evento desencadenante, debilidad y orina oscura o rojiza, de ahí que, ésta condición requiere una alta sospecha (54) para no postergar el tratamiento.

Los estudios habituales incluyen análisis metabólicos, CPK sérica, urianàlisis, análisis de enzimas intracelulares (GOT/ASAT, GPT/ALAT), LDH, creatinina, ùrea (hiperuricemia), ácido úrico y aldolasa (aunque no es sensible para la evaluación exclusiva de la rabdomiólisis ya que altas concentraciones son halladas también en cerebro e hígado).

En cuanto a los electrolitos pueden encontrarse alteraciones electrolíticas como: hiperpotasemia, hiperfosfatemia, hiperkalemia, haciendo necesario el monitoreo y la evaluación de los cambios cada 4 a 6 horas y así ver o no la necesidad de realizar diálisis(55), el peritaje de las alteraciones hematológicas y complicaciones renales puede prevenir daños mayores e incluso la muerte (56).

La medición de las concentraciones de creatincinasa es un método más sensible que la determinación de la mioglobina para establecer el diagnóstico de rabdomiolisis ya que al emprender el entrenamiento muchos individuos cursan con elevación de la mioglobina pero no en todos se manifiesta clínicamente (57), debe tenerse presente que los niveles de mioglobina ascienden más rápido que los de $\mathrm{CPK}$; Berman en 1977 mencionó que aunque la mio-hemoglobinuria es significativa lo realmente trascendental es la hemolisis intravascular subyacente (58).

En las rabdomiólisis de importancia clínica $>20.000$ a $50.000 \mathrm{UI} / \mathrm{L}$ de creatina cinasa sérica) la concentración sérica de creatinina se encuentra desmedidamente elevada respecto del BUN. La hiperkalemia severa suele aparecer en los primeros días post episodio, particularmente en los pacientes oligùricos. La elevación de la $\mathrm{CPK}$ representa un marcador excelente de esta enfermedad, y es medida con facilidad, algunas publicaciones hablan de la inmediatez de la aparición sérica, otras refieren aparición a partir de las 212 horas de la injuria muscular, llegando a su pico máximo entre las 24-72 horas (59). La insuficiencia renal aguda también puede presentarse con cifras inferiores a 5000 de $\mathrm{CPK}$, si conjuntamente hay acidosis, sepsis y deshidratación.

La mioglobinúria (60) por rabdomiólisis, conduce a necrosis tubular aguda, por lesión tubular proximal, los pacientes suelen cursar con glucosuria moderada y transitoria incluso sin presentar hiperglucemia.

La hiperfosfatemia se debe a la salida iónica del miocito causando precipitación de fosfato de calcio en los vasos sanguíneos, ojos y tejidos blandos, estimulando indirectamente la producción de hormona paratiroidea, en este trastorno el hueso es resistente a los efectos de la hormona, por lo que la hipocalcemia persiste. La hipocalcemia aparece tempranamente y se aprecia completamente después de las 24 horas iniciales.

\section{Recomendaciones terapéuticas}

El tratamiento se orienta a acelerar la recuperación, identificar y corregir cualquier causa reversible de la injuria renal (61) y muscular, prevenir y tratar las complicaciones como la insuficiencia renal (62); en caso de presentarse debe corregirse temprana y poderosamente la volemia ya que los pacientes con rabdomiólisis frecuentemente cursan con hipovolemia secundaria al edema muscular. Durante el tratamiento inicial generalmente se requieren 2 a $3 \mathrm{~L}$ de cloruro de sodio $0.9 \%$ por hora y al alcanzar la estabilidad hemodinámica entre 300 a $500 \mathrm{~mL}$ por hora, algunos autores argumentan que esta infusión de cristaloides por si solos pueden producir suficiente diuresis de solutos y prevenir la progresión a falla renal, algunas publicaciones mencionan que el uso adicional de manitol para impedir la precipitación y aumentar el aclaramiento de mioglobina en el riñón son útiles (63), sin embargo otras mencionan que el uso de manitol ó de bicarbonato no proporcionan beneficios adicionales con respecto a otro tipo de reposición agresiva de líquidos (64), sin embargo reportes de casos, estudios clínicos retrospectivos y estudios perpetrados en animales hablan sobre la superioridad de la alcalinización urinaria en animales, ya que la precipitación del complejo de la proteína de Tamm-Horsfall-mioglobina se encuentra aumentada en la orina ácida(65), por lo que adicionar $44-50 \mathrm{meq}$ de bicarbonato de sodio por litro de solución infundida prevendría probablemente la insuficiencia renal aguda al conseguir el $\mathrm{pH}$ urinario mayor a 6.5 y $\mathrm{pH}$ arterial de 7,457,5 . De igual manera el bicarbonato favorece la creación de orina alcalina, evitando la formación de cilindros y reduciendo el efecto toxico de la mioglobina. El objetivo es lograr diuresis de por lo menos 1,5-2,0 ml/kg/hora. Inhibiendo el ciclo redox tanto de la hemoglobina como de la mioglobina (66), al igual que la peroxidaciòn lipídica.

También ha sido descrito que el uso de manitol puede considerarse para mantener flujos urinarios aceptables, en dosis de $25 \mathrm{gr}$ iniciales en adultos y en niños de $0,5 \mathrm{gr}$ a 1 $\mathrm{gr} / \mathrm{kg}$, cuidando de no incurrir en hipovolemia; el manitol permite la eliminación de los tóxicos por los túbulos renales (67), mediante la creación de un gradiente que extrae el 
líquido acumulado en los músculos, mejorando la hipovolemia, y aliviando la presión de los compartimientos; dosis mayores a $200 \mathrm{~g} /$ día son vinculadas con insuficiencia renal aguda por nefrosis osmótica (68).

Es discutida la utilización de antioxidantes que disminuyan el daño directo de la mioglobina en el túbulo renal (69), la acetilcisteina es un antioxidante que contiene un tiol con muy poca toxicidad, y cuyo mecanismo de acción no ha sido esclarecido, aparentemente ofrece muy poco daño y un potencial beneficio, una buena estrategia es administrar 600 mg vía oral cada doce horas en pacientes con riesgo de presentar insuficiencia renal aguda, sobre todo si se van a usar medios de contraste para las ayudas imagenológicas. En todo caso las dosis de suministro de los medicamentos deben ajustarse de acuerdo con el grado estimado de deterioro renal, para fármacos que se eliminan por el riñón.

Deben tomarse medidas preventivas para evitar el exceso de volumen y la hiperpotasemia mediante el uso de diuréticos de asa (son los únicos que consiguen kaliuresis efectiva) a dosis altas: furosemida a dosis desde $20 \mathrm{mg}$ hasta $160 \mathrm{mg}$ vía oral o endovenosa dos veces al día para producir diuresis adecuada, no obstante algunos autores desalientan el uso de los diuréticos, pues, si bien convierten una insuficiencia renal oligúrica en no oligúrica disminuyendo la obstrucción tubular (70), en algunos pacientes no ofrecen recuperación; otra alternativa son los agentes que aumentan el pasaje de potasio del espacio extracelular al intracelular tales como la glucosa hipertónica y el bicarbonato (efectividad temporal), los bloqueantes intestinales de potasio o la diálisis.

Los efectos de la sobredosificación de los diuréticos son la sordera, por lo que la recomendación es iniciar con dosis de 0.4 a $0.6 \mathrm{mg} / \mathrm{kg} / \mathrm{h}$ de furosemida, incrementando la dosis hasta máximo $1 \mathrm{mg} / \mathrm{kg} / \mathrm{h}$. Los diuréticos tiacídicos endovenosos pueden usarse también para incrementar la producción de orina (clorotiacida 500 mg vía endovenosa cada 8 a 12 horas).

En pacientes con rabdomiólisis no se debe tratar la hipocalcemia a menos que sean sintomáticos, la hipocalcemia y la hiperfosfatemia pueden mejorar con dieta y agentes fijadores de fosfato, como el hidróxido de aluminio (500 mg orales con los alimentos) a corto plazo y el carbonato de calcio 500-1500 mg orales tres veces al día (el quelante de calcio debe usarse con precaución pues el aumento de calcio puede aumentar la precipitación de fosfato en el músculo lesionado), acetato de calcio (667 mg, 2 o 3 tabletas, oral después de los alimentos) o sevelamer (800 a $1600 \mathrm{mg}$ oralmente, tres veces al día). Puede coexistir hipermagnasemia debido a la menor excreción de magnesio por los túbulos renales; por lo que se hace mandatorio coartar el uso de antiácidos y laxantes con esta composición.

\section{Recomendaciones para el manejo de un ataque agudo}

- Hospitalizar al paciente para controlar los síntomas y realizar la evaluación hidroelectrolítica, las irregularidades electrolíticas acaecidas por la IRA inducida por Rabdomiólisis deben recibir tratamiento inmediato, particularmente la hiperkalemia.

- Interrogar sobre el uso de fármacos y medicamentos enrojecedores de orina: moras, remolachas, ruibarbos, colorantes para alimentos, habas, fenolftaleína, rifampicina, doxorrubicina, deferoxamina, cloroquina, ibuprofeno y metildopa, también sobre los que tiñen la orina de color marrón: levodopa, metronidazol, nitrofurantoina, hierro, sorbitol y cloroquina.

- Establecer diagnósticos diferenciales frente a hemorragia en el tracto urinario inferior: cistitis aguda, cálculos, tumores ureterales; ò traumatismo; lesión, infección, estrecheces y carcinomas en la vejiga; cistitis por radiación o carúnculas ureterales.

- Sospechar la etiología de la eritrocituria, recordar que puede deberse a enfermedades renales, glomerulonefritis, hipertensión maligna, poliquistosis renal, nefritis lùpica, infarto renal, nefrosclerosis maligna, infecciones renales agudas, tumores renales, trombosis de la vena renal, glomerulonefritis crónica, tuberculosis renal, periuretritis, síndrome nefrótico, necrosis papilar aguda, hidronefrosis y como en este caso debido a daño glomerular por nefrotoxinas endógenas: mioglobina.

- Indagar sobre el padecimiento de leucemia, trombocitopenia, déficit de factores de coagulación, drepanocitosis o rasgo drepanocítico, desnutridos con escorbuto. Los anticoagulantes son también agentes etiológicos. La nefritis intersticial aguda o la cistitis secundaria al uso de penicilinas y cefalosporinas también deben tenerse en cuenta.

- Proporcionar apoyo nutricional para evitar el catabolismo excesivo, prevenir la acidosis metabólica mediante la restricción de proteínas dietéticas a $0.6 \mathrm{~g} / \mathrm{kg} /$ día.

- Usar medicamentos con efectividad comprobada en rabdomiolisis.

- Infundir líquidos intravenosos en forma generosa, teniendo en cuenta la aparición de signos de sobrecarga.

Usar antioxidantes, pentoxifilina, vitaminas E y C como barrenderos de radicales libres, estos son empleados en el tratamiento y prevención de la Insuficiencia renal aguda mioglobinúrica, aunque hoy por hoy falten estudios controlados que certifiquen su uso, tampoco hay estudios que lo contraindiquen. 


\section{Seguimiento y prevención}

Educar al paciente, la familia y los entrenadores deportivos sobre la enfermedad, los factores precipitantes y la adecuada hidratación, el reposo post ejercicio, la nutrición adecuada, y los descansos inter ejercicio como factores protectores. En caso de que al paciente se le establezca componente hereditario, se debe direccionar a asesoría genética.

Dado que la cantidad de sudor raramente se compensa con los líquidos ingeridos en individuos con dificultad para la hidratación como militares, soldados, atletas y trabajadores industriales (71), vale la pena adoptar las recomendaciones que el Colegio Americano de Medicina Deportiva, publicó sobre la hidratación y nutrición previas al ejercicio (72):

- Consumir una dieta balanceada y mantener la hidratación normal por lo menos hasta 24 horas antes del evento o entrenamiento. Hidratación temprana y constante.

- Consumir dos horas antes del evento entre 16 a 20 onzas (400-600 ml) de agua o bebidas deportivas garantizando tiempo para la excreción antes de la actividad, de igual modo, consumir entre 7 a 10 onzas cada 10-20 minutos durante la carrera (600-1680 $\mathrm{ml} / \mathrm{h})$.

- Aunque el consumo de líquido está sujeto a tolerancia, el deportista no debe esperar a sentir sed para comenzar a hidratarse

- Para actividades en condiciones extremas, superiores a 50 min o más, se deben beber líquidos que proporcionen sustrato energético muscular.

- Para actividades que superen la hora de intensidad se debe adicionar sodio, para facilitar así la retención hídrica y prevenir hiponatremia, el sodio es el mayor catión del espacio extracelular, pero igualmente el mayor electrolito presente en el sudor. La cantidad de sodio en el sudor se estima entre 20 a $80 \mathrm{mmol} / \mathrm{l}$. Por lo que la concentración aproximada para el reemplazo de sodio en los líquidos oscilaría entre 0.5 a $0.7 \mathrm{~g} / \mathrm{L}$ (73).

- Para la rehidratación post ejercicio se aconseja consumir entre 20 y 40 onzas $(560-800 \mathrm{ml})$ de líquido o si se rehidrata con bebida deportiva ingerir en proporción a cada libra de peso perdida durante el ejercicio.

- Adicionalmente se recomienda ingerir $250 \mathrm{ml}$ de líquidos en lapsos de 15-20 minutos de actividad física (74).

- Los electrolitos en las bebidas deportivas reducen el riesgo de calambres musculares, fatiga y alteraciones electrolíticas, así mismo disminuyen el riesgo de intoxicación hídrica, tras largas y acaloradas jornadas. Adicionar potasio reduce el volumen urinario y promueve la retención hídrica. Por mencionar un ejemplo de la efectividad del consumo de bebidas con electrolitos frente al agua, en enero del 2002 el instituto científico de deportes Gatorade ${ }^{\circledR}$ en "Runners World North Americas" bajo el encabezado "Directriz de líquidos" mencionó que Gatorade ${ }^{\circledR}$ remplaza $42 \mathrm{mmol}$ respecto a los $0 \mathrm{mml}$ que hace el agua y que de igual manera la osmolaridad plasmática cae cerca del $12 \%$ en los bebedores de agua respecto a los consumidores de Gatorade ${ }^{\circledR}$.

- La preparación del deportista debe ser en ambientes frescos, antes de realizar ejercicios en ambientes cálidos, se prefieren las horas de la mañana y de la noche para las intensidades más altas de ejercicio y las horas calurosas del día se pueden usar para ejercicios no tan intensos.

\section{Pronóstico}

La mortalidad estimada es de $8-15 \%$ en pacientes con mioglobinuria secundaria a rabdomiólisis en ausencia de insuficiencia renal y aumenta hasta $42-51 \%$ cuando la IRA aparece (75).

\section{Discusión}

Al indagar por reportes similares en Colombia, no se encontraron datos epidemiológicos de rabdomiólisis, de igual modo no hay reportes bibliográficos recientes. En cuanto a la incidencia, se aproxima que será más frecuente observar ésta condición dado el incremento de ejercicios intensos en instituciones ò entrenamientos individuales, aunado al uso de sustancias no prescritas para la realización del deporte e hidratación ineficiente.

En éstos pacientes las causas que pudieron precipitar la rabdomiólisis son multifactoriales y hay evidencia del comportamiento clínico de la patología manifestada por emisión de orina rojo-achocolatado, debilidad muscular y mialgias. Los pacientes recibieron hidratación abundante con solución salina endovenosa a $2 \mathrm{~L}$ inicialmente $y$ posteriormente se continuó la infusión a $200 \mathrm{ml} / \mathrm{h}$ hasta obtener orina clara, este proceso fue llevado durante un tiempo aproximado de 5 días, para cada uno; en el paciente 1 de manera precoz se inició alcalinización de la orina por un periodo de 2 días, mediante la adición de $50 \mathrm{meq}$ de bicarbonato de sodio por cada litro de solución administra$\mathrm{da}$, manteniendo el pH arterial en los rangos aceptados $(5,45$ a 7,50), en el paciente 2 el tratamiento fue llevado a cabo con infusión endovenosa de solución salina al $0.9 \%$ exclusivamente, hasta evidenciarse orina clara, en ambos casos la evolución indicó mejoría con disminución de enzimas musculares y no se requirió diálisis.

Con un reporte de 2 casos clínicos no se puede considerar el inicio de actividad física como factor exclusivamente 
implicado en la rabdomiólisis, son múltiples las situaciones causantes de citólisis muscular y en éstos pacientes presentados faltaron pruebas de contracción muscular, análisis bioquímico del músculo, resonancia magnética nuclear espectroscópica con fosforo 31, para investigar miopatías almacenadoras de lípidos, miopatías mitocondriales, desordenes del metabolismo oxidativo o sensibilidad a la hipertermia maligna.

En todo caso son destacables las recomendaciones que se realizan para aminorar la injuria muscular, tomar medidas preventivas y hacer diagnóstico temprano teniendo suspicacia clínica en los grupos con algún tipo de predisposición o factor facilitador de lisis muscular.

\section{Conclusiones}

- En los pacientes se evidenció el cuadro típico de rabdomiólisis por esfuerzo, en ambos los síntomas de afectación muscular y la elevación de las enzimas musculares, fueron clásicos presentando orina rojiza, en ellos no hubo oliguria ni fue necesaria la realización de hemodiálisis. El manejo fue conservador, con aporte adecuado de solución salina y alcalinización urinaria con bicarbonato en el paciente uno (1). La función renal se recuperó y actualmente los pacientes están asintomáticos.

- Teniendo en cuenta la intensidad del entrenamiento a la que se exponen individuos con demandas físicas altas (atletas, soldados), y la tendencia a extenuantes jornadas de ejercicios para "mantenerse en forma" los casos de rabdomiólisis aumentaran en reporte, de ahí la importancia de tomar medidas preventivas, y agudizar la sospecha diagnostica ante un paciente con la sintomatología sugestiva.

- La rehidratación oportuna es beneficiosa en el deportista ya que incrementa el flujo sanguíneo, reduce la hiperosmolaridad, reduce la deshidratación celular y ayuda al mantenimiento del fluido extravascular.

- Las prendas algodonadas además de adicionar peso al deportista, impiden la transpiración y la evaporación eficiente del sudor, además de provocar excoriaciones cutáneas, ampollas e infecciones en piel, condiciones ampliamente presentadas en los deportistas, por lo que se estimula el uso prendas con poliéster (material liviano, transpirable, suave y flexible) y nailon absorbente, durante la actividad deportiva, para facilitar la transpiración y permitir la rápida evaporación del sudor de la piel, manteniendo fresco al atleta. Por la composición de las prendas con que realizaron ejercicio éstos pacientes, se dedujo que pudieron favorecer la hipertermia durante el entrenamiento.

- Antes, durante y posterior a la actividad física, la hidratación es crucial. En los pacientes evidentemente fue ineficiente. El consumo del líquido está supeditado al acceso económico, al suministro limitado y a los intervalos de descanso durante el entrenamiento.

- La rabdomiólisis debe buscarse intencionalmente en los pacientes con historia de esfuerzo físico intenso, asociado con dolor muscular.

- Es necesario crear protocolos estrictos de rehidratación en todas las poblaciones vulnerables.

\section{Agradecimientos}

Al doctor Héctor Hugo Otálora Parra, médico Internista y Gastroenterólogo, al doctor Jorge Humberto Castillo Silva, médico Fisiatra, epidemiólogo y Doctor en Educación, por sus valiosos aportes, orientaciones y revisiones.

\section{Conflictos de interés}

Los autores declaran no tener de manera directa o indirecta, ningún tipo de conflicto de intereses financieros, académicos o laborales que puedan poner en peligro la validez de este estudio.

\section{Financiación}

Este trabajo se realizó con apoyo financiero exclusivo del autor.

\section{Referencias}

1. Holt S, Moore K. Pathogenesis of renal failure in rhabdomyolysis: The role of Myoglobin. Exp Nephrol. 2000;8:72-76

2. Vanholder R, Severe MS, Erek E, Lameire N. Rhabdomyolysis. J Am Nephrol. 2000;11:1553-1561.

3. Ho AM, Karmakar MK, Contardi LH, Ng SS, Hewson JR. Excessive use of normal saline in managing traumatized patients in shock: a preventable contributor to acidosis. $J$ Trauma 2001;51(1):173-177.

4. Lieberthal W, Levinsky N. Treatment of acute tubular necrosis Semin Nephrol 1990;10:571-583.

5. Homsi E, Barreiro M, Orlando J, Higa E. prophylaxis of acute renal failure in patients with rhabdomyolysis. Ren Fail 1997,19:289-288.

6. Tein I, Dimauro S, Devivo D. Recurrent childhood myoglobinuria. Adv Pediatr. 1990;37:77.

7. Tonin P, Lewis P, Servidei S, Dimauro S. Metabolic causes of mioglobinuria. Ann Neurol. 1990;27:181-185.

8. Dougal W, Gary G, Jay D. Exercise Rhabdomyolysis in Military Aircrew: Two Cases and a Review of Aeromedical Disposition. Clin Med. 2000;71(11):1140.

9. Poels PJE, Gabreëls FJM. Rhabdomyolysis: a review of literature. Clin Neurol Neurosurg. 1993;95:172-92.

10. Henares Garcia P. Rabdomiolisis secundaria a ejercicio físico en un gimnasio. Semergen. 2012;38:53-55.

11. Schwaber M, Liss H, Steiner I, Brezia M. Hazard of sauna use after strenuous exercise. Ann Intern Med. 1994;120:44.

12. Olerud J, Homer L, Carroll H. Incidence of acute exertional rhabdomyolysis. Serum myoglobin and enzyme levels as indicator of muscle injury. Arch Intern Med. 1976;136:692697. 
13. Ward MM. Factors predictive of acute renal failure in rhabdomyolysis. Arch Intern Med. 1988;148:1553-1557.

14. Brivet FG, Kleinknecht DJ, Loirat P, Landais PJ. Acute renal failure in intensive care units- causes, outcomes, and prognostic factors of hospital mortality: a prospective multicenter study. French Study Group on Acute Renal Failure. Crit Care Med. 1996;24:192-198.

15. Splendiani G, Mazzarella V, Cipriani S, Zazzaro D, Casciani C; Dialytic treatment of rhabdomyolysis-induced acute renal failure: our experience. Ren Fail. 2000;23:183-191.

16. Odeh $\mathrm{M}$. The role of reperfusion-induced injury in the pathogenesis of the crush syndrome. $\mathrm{N}$ Engl J Med. 1991;324:1417-1422.

17. Knochel J. Mechanisms of rhabdomyolysis. Curr Opin Rheumatol. 1993;5:725-731.

18. Office of the surgeon general department of the army, United States of America. Rhabdomyolysis and compartment syndrome in military trainees. In: Government Printing Office, editor. Recruit Medicine. 2006;p.167.

19. Carcelìn-Garza JR, Dìa-Gutièrrez S. Rabdomiólisis. Comunicación de dos casos relacionados con el esfuerzo y revisión de la bibliografía. Med Int Mex. 2013;29:410-423.

20. Zager RA, Foerder CA. Effects of inorganic iron and myoglobin on in vitro proximal tubular lipid peroxidation and cytotoxicity. J Clin Invest. 1992;89(3):989-995.

21. Greenhill A, Gruskin AB. Laboratory evaluation of renal function. Pediatr Clin North Am. 1976;23(4):661-679.

Golab F, Kadkhodaee M, Zahmatkesh M, Hedayati M, Arab M Schuster R, Zahedi K, Lentsch AB, Soleimani M. Ischemic and non-ischemic acute kidney injury cause hepatic damage. Kidney Int. 2009;75(8):783-792.

23. LiX, Hassoun Ht, Santora R, Rabb H. Organ crosstalk: the role of the kidney. Curr Opin Crit Care. 2009;15(6):481-487.

24. Grigoryev DN, Liu M, Hassoun HT, Cheadle C, Barnes KC, Rabb H. The local and systemic Inflammatory Transcriptome after Acute kidney Injury. J Am Soc Nephrol [Revista on-line]. 2008[consultado 05 enero 2014];19(3)547-558. Disponible en: http: //www.ncbi.nlm.nih.gov/pmc/articles/ PMC2391061/

25. Hayes LD, Morse CI. The effects of progressive dehydration on strength and power: is there a dose response?. Eur J Appl Physiol. 2010;108:701-707.

26. Eiser AR, Swartz CH . The Effects of exercise on Genitourinary Function. In: Bove AA, Lowenthal DT, editors. Exercise Medicine: Physiological Principles and Clinical Applications. Academic press; 1983; p.109.

27. Martínez MM, Pastor VJ, Sendra PF. Termoterapia. En: Manual de Medicina Física. 1a ed. Barcelona: Harcourt Brace de España. 1998.p.73-78.

28. Goulet EDB. Effect of exercise-induced dehydration on time trial exercise performance. A meta-analysis. Br. J. Sports Med. 2011;45:1149-1156.

29. Singh P, Okusa MD. The role of tubuloglomerular feedback in the pathogenesis if acute kidney injury. Contrib Nephol. 2011;174:12-21.

30. Garnès RA, Hernandez RR, Molina MJ, Masià PO. Rabdomiolisis en deportista veterano. 2008;43(160):189191.

31. Richter E, Turcotte L, Hespel P, Kiens B. Metabolic responses to exercise: Effects of endurance training and implications for diabetes. Diabetes Care. 1992; 15(11):1767-1776.

32. Nadel ER. Circulatory and thermal regulations during exercise. Fed Proc. 1980;39(5):1941-1947.

33. Costill DL, Dalsky GP, Fink WJ. Effects of caffeine ingestion on metabolism and exercise performance. Med Sci Sports. 1978;10:155-58.

34. Tavares C, Kimiko R. Cafeína para el tratamiento del dolor. Rev Bras Anestesiol. 2012;62(3):387-401.
Palacios GA, Gutierrez EI, Ùbeda MN, efecto de la cafeína en el rendimiento deportivo. Med Clin (Barc). 2008;131:751-755. Armstrong LE. Caffeine, body fluid electrolyte balance, and exercise performance. Int $\mathrm{J}$ Sport Nutr Exerc Metab. 2002;12:189-206.

37. Maughan RF, Griffin J. Caffeine ingestion and fluid balance: a review. J Hum Nutr Diet. 2003;16:411-420.

38. Grandjean AJ, Reimers KJ, Bannick KE, Haven MC. The effect of caffeinated, non-caffeinated, caloric and non-caloric beverages on hydration. J Am Coll Nutr. 2000;19:591-600.

39. Roti MW, Casa DJ, Pumerantz AC, Watson G, Judelson DA, $\mathrm{Diaz} \mathrm{JC}$, et al. Thermoregulatory responses to exercise in the heat: chronic caffeine intake has no effect. Aviat Space Environ Med. 2006;77:124-120.

40. Wemple RD, Lamb DR, McKeever KH. Caffeine vs Caffeinefree sports drinks: effects on urine production at rest and during prolonged exercise. Int J Sports Med. 1997;18:40-46.

41. Costantini N, Hackney AC. Hormonal Regulation of Fluid and Electrolyte Homeostasis During Exercise. In: Conn PM,editors. Endocrinology of physical activity and Sport. New York. Humana Press; 2013.p.229.

42. Casa DJ, Armstrong LE, Hillman SK, Mountain SJ, Reiff RV, Rich BS, Roberts WO, Stone JA. National athletic trainer`s association position statement: fluid replacement for athletes. J Athl Trai. 2000; 35(2): 212-224.

43. Davis Jm, Jackson Da, Broadwell MS, Query JL, Lambert CL. Carbohydrate drinks delay fatigue during intermittent, high intensity cycling in active men and women. Int $J$ Sport Nutr. 1997;7:261-273.

44. Maughan RJ, Merson SJ, Broad NP, Shirreffs. Fluid and electrolyte intake and loss in elite soccer players during training. Int J Sport Nutr Exerc Metab. 2004;14(3):333-346.

45. Park SG, Bae YJ, Lee YS, Kim BJ. Effects of rehydration fluid temperature and composition on body weight retention upon voluntary drinking following exercise-induced dehydration. Nutr Res Pract. 2012;6(2):126-131.

46. Rehrer NJ. Fluid and electrolyte balance in ultra-endurance sport. Sports Med. 2001;31:701-715.

47. Iriarte C. La fuerza. Entrenamiento para la salud y la estética. Grupo Sobre Entrenamiento; número 42.

48. Cappa D. Principales problemáticas del entrenamiento de la potencia muscular. Entrenamiento de la potencia muscular. Mendoza-Argentina: Grupo Sobre Entrenamiento; 2000.p.10-11.

49. Vanholder R, Sevier M, Erek E, Lamerte N. Acute renal failure related to the crush sindrome: tomwards an era of seísmonephrology?. Nephrol Dial Transplant. 2000;15:1517.

50. Curtis BA. $\mathrm{Na} / \mathrm{Ca}$ exchange and first messenger $\mathrm{Ca}$ in skeletal muscle excitation-contraction coupling. Adv Exp Med Biol. 1992;311:1-17.

51. Montero J, Lovesio C, Godoy MV, Ruiz G. Rabdomiolisis por spinning en nueve pacientes. Medicina ( $B$ Aires).2009;69:153-156.

52. Knochel J, Schlein E. On the mechanism of rhabdomyolysis in potassium depletion. J Clin Invest. 1972;51:1750.

53. Knochel JP. Exertional rhabdomyolysis. N Engl J Med. 1972;287:463-472.

54. Llorca JA, Palomar M, Gàmez J, Bonell JM, y col. Rabdomiolisis idiopática. Med Intensiva 2001;25:161-163.

55. Pérez M, Roiz JC, Diazaraque R. Rabdomiolisis inducida por el ejercicio. MEDIFAM. 2001;11:562-565.

56. Line RL, Rust GS. Acute exertional rhabdomyolysis. Am Fam Physician. 1995;52(2):502-6.

57. Olerud J, Louis D, Carroll H. Incidence of acute exertional rhabdomyolysis. Serum myoglobin and enzyme levels as indicadors of muscle injury. Arch Intern Med.1976; 136(6):692-697.

58. Berman $\mathrm{lb}$. When the urine is red. J Am Med Assoc. 
1977;237:2753-2754.

59. Pèrez UM, Roiz FJ, Diazaraque MR. Rabdomiolisis inducida por el ejercicio. MediFam.2001;11:562-565.

60. Watson DB, Gary GW, Doucet JJ. Exercise Rhabdomyolysis in Military Aircrew: Two Cases and a Review of Aeromedical Disposition. Clin Med. 2000;71(11):1137-1141.

61. Blantz RC, Singh P. Analysis of the prerenal contributions to acute kidney injury. Contrib Nephrol. 2011;174:4-11.

62. Woodrow G, Brownth AM, Bruney JH. The clinical and features of acute renal failure due to rhabdomyolysis. Ren Fail. 1995; 17:467-474

63. Zager R. Studies of mechanisms and protective maneuvers in myoglobinuric acute renal injury. Lab Invest. 1989;60:619629.

64. Zager R: Combined mannitol and deferoxamine therapy for myohemoglobinuric renal injury and oxidant tibular stress. Mechanistic and therapeutic implications. J Clin Invest. 1992,90:711-719.

65. Heyman SN, Greenbaum R, Shina A, Rosen S, Brezis M, Myoglobinuric acute renal failure in the rat: a role for acidosis? Exp Nephrol. 1997;5(3):210-216.

66. Moore KP, Holt SG, Patel RP, Svistunenko DA, Zackert W, Goodier D, et. al. A causative role for redox cycling of myoglobin and its inhibition by alkalinisation in the pathogenesis and treatment of rhabdomyolysis-induced renal failure. J Biol Chem. 1998; 273(48):31731-31737.

67. Zager RA, Foerder C, Bredl C. The influence of mannitol on myoglobinuric acute renal failure: functional, biochemical, and morphological assessments. J Am Soc Nephrol. 1991;2(4):848-855.

68. Visweswaran P, Massin EK, Dubose TD Jr. Mannitol-induced acute renal failure. J Am Soc Nephrol. 1997;8(6):1028-1033.

69. Nath KA, Balla J, Croatt AJ, Vercellotti GM. Heme Proteinmediated renal injury, a protective role for 21 -aminosteroids in vitro an in vivo. Kidney Int 1995;47:592-602.

70. Kellum JA. The use of diuretics and dopamine in acute renal failure: a systematic review of the evidence. Crit Care. 1997;1(2):53-59.

71. Schrier RW, Henderson HS, Tisher CC, Tannen LT. Nephropathy associated with heat stress and exercise. Ann Intern Med 1967;67(2):356-376

72. American College of Sports Medicine, Sawka MN, Burje LM, Eichner ER, Maughan RJ, Montain SJ, et al. American College of Sports Medicine position stand. Exercise and fluid replacement. Med Sci Sports Exerc. 2007; 39(2):377-390.

73. Meyer F, Bar-Or O, Salsberg A, Passe D. Hypohydration during exercise in children: effect on thirst, drink preferences, and rehydration. Int J Spors Nutr. 1994;4(1):22-35.

74. Costill DL. Gastric emptying of fluids during exercise. In: Gisolfi CV, Lamb DR, eds. Perspectives in exercise science and sports medicine. Vol 3. Fluid homeostasis during exercise. Carmel, Indiana: Benchmark Press, 1990:97-121.

75. Fernández Funez A, De Tomàs E, Alamillo A, Puras A. Rabdomiolisis no traumática, etiología y factores preventivos de insuficiencia renal aguda. Med Clin (Barc). 1995;105:412415. 